\title{
Classicalization of gravitons and Goldstones
}

\author{
Gia Dvali, ${ }^{a, b, c, d}$ Cesar Gomez ${ }^{a, e}$ and Alex Kehagias ${ }^{f}$ \\ ${ }^{a}$ Arnold Sommerfeld Center for Theoretical Physics, Department für Physik, \\ Ludwig-Maximilians-Universität München, \\ Theresienstr. 37, 80333 München, Germany \\ ${ }^{b}$ Max-Planck-Institut für Physik, \\ Föhringer Ring 6, 80805 München, Germany \\ ${ }^{c}$ Theory Division, CERN, \\ 1211 Geneva 23, Switzerland \\ ${ }^{d}$ CCPP, Department of Physics, New York University, \\ 4 Washington Place, New York, NY 10003, U.S.A. \\ e Instituto de Física Teórica UAM-CSIC, C-XVI, Universidad Autónoma de Madrid, \\ Cantoblanco, 28049 Madrid, Spain \\ ${ }^{f}$ Physics Division, National Technical University of Athens, \\ 15780 Zografou Campus, Athens, Greece \\ E-mail: Georgi.Dvali@cern.ch, bfkl08@gmail.com, \\ kehagias@central.ntua.gr
}

ABSTRACT: We establish a close parallel between classicalization of gravitons and derivatively-coupled Nambu-Goldstone-type scalars. We show, that black hole formation in high energy scattering process represents classicalization with the classicalization radius given by Schwarzschild radius of center of mass energy, and with the precursor of black hole entropy being given by number of soft quanta composing this classical configuration. Such an entropy-equivalent is defined for scalar classicalons also and is responsible for exponential suppression of their decay into small number of final particles. This parallel works in both ways. For optimists that are willing to hypothesize that gravity may indeed selfunitarize at high energies via black hole formation, it illustrates that the Goldstones may not be much different in this respect, and they classicalize essentially by similar dynamics as gravitons. In the other direction, it may serve as an useful de-mystifier of via-blackhole-unitarization process and of the role of entropy in it, as it illustrates, that much more prosaic scalar theories essentially do the same. Finally, it illustrates that in both cases classicalization is the defining property for unitarization, and that it sets-in before one can talk about accompanying properties, such as entropy and thermality of static classicalons (black holes). These properties are by-products of classicalization, and their equivalents can be defined for non-gravitational cases of classicalization.

KEywords: Solitons Monopoles and Instantons, Nonperturbative Effects, Black Holes ARXIV EPRINT: 1103.5963 


\section{Contents}

1 Introduction 1

2 Classicalization of Goldstones 4

$\begin{array}{lll}3 & \text { Classicalization by gravitons } & 7\end{array}$

4 Classicalization of graviton scattering $\quad 10$

5 Parallels and differences $\quad \mathbf{1 2}$

5.1 Classicalons in gravity versus Goldstone $\quad 12$

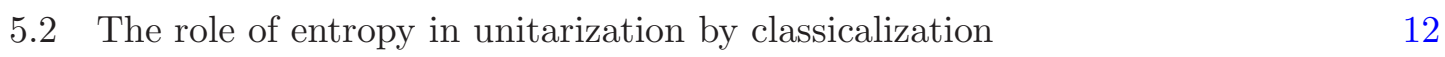

5.3 If not $N \rightarrow 2$, then why $2 \rightarrow N$ ? 13

6 Classicalization landscape: gravity as a most efficient classicalizer $\quad \mathbf{1 5}$

$\begin{array}{ll}\text { 6.1 Graviton as a most efficient classicalizer } & 15\end{array}$

$\begin{array}{ll}\text { 6.2 Generalized holographic bound on information storage } & 19\end{array}$

6.3 Black hole entropy as classicalon entropy? 20

$\begin{array}{lll}7 & \text { Conclusions } & 21\end{array}$

\section{Introduction}

Recently, a concept of non-Wilsonian UV-self completeness for a class of non-renormalizable theories was introduced in [1-3]. The meaning of this concept is, that a seemingly unitaryviolating theory prevents us from going to sub-cutoff distances, by becoming classical in deep UV. This concept was inspired by the original suggestion for Einstein gravity $[4,5]$, due to generically-expected feature, that high-energy collisions there produce black holes. It was suggested, that this fact leads us to self-completeness since black hole formation automatically prevents us from probing sub-Planckian distances. Inspired by this property of gravity, in [1] this concept of self-UV-completeness, which we refer to as classicalization, was generalized to a class of derivatively-self-interacting theories. Defining property of such theories is energy self-sourcing and existence of classical radius that sets the interaction range at high center of mass energy and dominates over all the relevant quantum length-scales.

Satisfactory understanding of physical viability of the classicalization phenomenon requires field-theoretic analysis based on combination of perturbative and non-perturbative methods. Subsequent studies $[2,3]$ based on non-perturbative analysis of scattering process, confirm the emergence of the classical length scale in such processes. In what follows, we shall also employ such analysis. A promising approach in this direction would be to develop a path integral formulation of the problem, as suggested recently in [6]. 
The main purpose of the present work is to establish a deeper connection between classicalization phenomena in gravity and in Goldstone-type scalar theories, for the sake of improving understanding on both sides.

The idea of classicalization, of course, raises number of important question, one of which is, how close are the parallels between gravity and Goldstones?

In other words there are two major questions.

1) Do black holes really unitarize scattering amplitudes, and if yes, why? and

2) How special are black holes as compared to other classical configurations for unitarization of the amplitudes?

These questions are closely related and (as we shall see) the answer to the first question is also a key for answering the second one. In order to outline our claims and findings, let us deconstruct the above questions.

The known properties of the black holes are: 1) (semi)classicality; 2) classical stability; 3) existence of Schwarzschild (or other) horizon; and 4) the subsequent properties: Absence of classical hair, existence of Hawking temperature and of Bekenstein-Hawking entropy.

All the properties listed in 4) are the consequences of 1)-3). But obviously, the property 1) (and 2)) can exist without the rest. In order to understand which of these properties are crucial for unitarization, let us outline why we even expect that black holes can unitarize high energy scattering amplitudes.

The idea is simple. In order to probe short distances one needs to do high-energy scattering with small impact parameter. This requires bringing a lot of energy within a small volume. Because any form of energy sources gravity, bringing a lot of energy within the small volume creates a classical gravitational field. If volume is small (equivalently if energy is large) so that energy concentration is within its Schwarzschild radius $r_{g}$, the whole region is expected to collapse into a black hole [7]. The inevitability of the very last step for the case of arbitrary topology is still under debate, but we shall not enter there. Our findings are precisely directed to bypass this issue and reduce the unitarization process to its bare essentials (for which, as we shall argue, what matters is not a "blackholeness" but rather classicality). So therefore, let us pick up a spherically-symmetric geometry in which case most of us probably would agree that black hole formation is inevitable.

Lets us note here that understanding the high-energy scattering in gravitational theories is a central problem and has attracted a lot of attention both in the framework of Einstein gravity as well as in supergravity and/or string theory [8]-[18, 19]. An emerging feature in all these studies, is that quantum gravity is endowed with deep long-distance issues connected with black hole formation. The latter provides a classical scale, the Schwarzschild radius, larger than any quantum scale, and seems to lead to the conclusion that unitarity in gravity theory is not a short-distance problem and cannot be resolved by any UV modification of the theory. For example, string theory has improved perturbation theory compared to QFT, which however, breaks down precisely at impact parameter of the order of Schwarzschild radius. Although there is no full proof for the inevitability of black hole 
formation in transplanckian scattering with sub-Schwarzschild radius impact parameter, there are indications by numeric simulations for example [20], that this is indeed the case. In addition, the radiated away gravitational energy has been calculated in [21, 22] and it is a finite fraction of the total center of mass energy $\sqrt{s}$ forming possibly a large black hole with mass $\sim \sqrt{s}$. It should be also noted that in 2+1-dimensions the inevitability of black hole formation has been demonstrated for head-on collision [23].

Now, once black hole is formed, its decay into any few-particle quantum state is exponentially suppressed. Whatever property is behind this suppression, is the key to the unitarization process. For the black holes this suppression can be understood from any of the properties 1-4) listed above. And in particular, the suppression can be easily understood from the properties of entropy and thermality, because probability to produce a pair of highly energetic particles with energy equal to an entire mass of a black hole $\left(M_{\mathrm{BH}}\right)$ in a thermal evaporation process at temperature $T_{H}^{-1} \sim M_{\mathrm{BH}} L_{P}^{2}$ is at least Boltzmann suppressed by a factor

$$
\Gamma_{\mathrm{BH} \rightarrow 2} \sim \mathrm{e}^{-\frac{M_{\mathrm{BH}}}{T_{H}}} \sim \mathrm{e}^{-\left(M_{\mathrm{BH}} L_{P}\right)^{2}} .
$$

Let us ask now, are all 1)-4) properties necessary for such a suppression? The answer to this question is negative. In fact property 1), classicality is the defining property. Once we understand that a given object is classical, the suppression of any two-particle decay automatically follows, regardless of entropy and any other property. In order to see this, let us imagine that in a scattering experiment we stop short of forming a horizon, but still form a classical configuration of the gravitational field of the size not that different from a Schwarzschild horizon of a would-be black hole $\left(r_{g}\right)$. Since, there is no horizon, such a configuration carries no entropy or temperature, but it is nevertheless classical.

How probable is the decay of such a classical configuration say in a two-particle quantum state? Of course, intuitively we understand that the suppression must be exponential, but in order to quantify it, let us analyze classicality from field-theoretic perspective. One of the signals of classicality is that the mass $M$ of a configuration is much larger than its inverse size $r_{*}^{-1}$. In other words, we deal with a coherent superposition of many soft quanta. The number of quanta can be estimated to be $N \sim M r_{*}$. Now the question is how probable is the decay of a coherent $N$-particle state into two quanta? This decay (up to factors of order $\log N$ in the exponent) is suppressed as,

$$
\Gamma_{N \rightarrow 2} \sim \mathrm{e}^{-N}
$$

Notice, that when applied to black holes, this counting correctly reproduces the above Boltzmann (or entropy) factor, but it is much more general. We thus learn, that neither entropy nor thermality are the defining universal suppression factor, but rather classicality.

It is classicality and not "blackholeness" the defining universal reason for unitarization!

Being liberated from the necessity of having entropy (or even a horizon), we can ask why can't other classical configurations that are also result of energy sourcing play the role in unitarizing the scattering cross sections? This is the key idea of classicalization. 
In fact, we can turn the above reasoning around, and argue that classicalon configurations allow us to define a notion of an entropy precursor, in terms of the number of soft quanta produced in classicalization process, which in the particular case of black holes agrees with the entropy counting, but is much more general and applicable to other classical configurations produced by energy self-sourcing. This view can also shed a different light at the origin of black hole entropy, by viewing it as a necessary outcome of classicalization, which requires production of many soft quanta, and not vice versa.

Classicalons share with black holes two properties that are crucial for unitarization. These are, the energy (self)sourcing and classicality. Thus, the bare essentials of unitarization process in these two different theories are surprisingly similar.

We hope, that a simple calculation below illustrates the viability and the depth of this connection. For this we shall consider the scattering process in gravity up to the point where we stop short of horizon formation, and show that formation of classical configuration is closely connected to the formation of analogous configuration in a scattering process involving self-sourcing of Goldstone-type scalars.

The idea is to repeat the calculation of the scattering process of [2], which was performed there for Goldstone waves, for a spin-2 system. Namely, we consider scattering of wave-packets with a very small occupation number but trans-Planckian center of mass energy and see how the system classicalizes at larger and larger distances. Not surprisingly we shall discover that the role of the classicalization radius, $r_{*}$, is played by the Schwarzschild radius corresponding to the center of mass energy.

This emerging parallels allow us to give an useful unified parameterization of the landscape of classicalizing theories, by parameterizing how efficiently the classicalization radius $r_{*}(s)$ grows with energy $\sqrt{s}$. We shall see, that gravity (spin-2 field) is a most efficient classicalizer, and gives a linear growth, $r_{*} \propto \sqrt{s}$. In all other cases, of spin-0 classicalizer fields, the growth is slower. This parameterization also allows to generalize the holographic bound on information storage to all classicalizing theories. The bound is the most stringent for gravity and non-existent for weakly-coupled non-classicalizing theories, with spin-0 classicalizing theories occupying intermediate states.

In order to try to achieve a maximal clarity, we shall structure our discussion in the following way. First, we shall briefly go through the essential ingredients for the Goldstone analysis, and give an universal definition of the $r_{*}$-radius. Then we shall repeat the similar analysis for gravitons. Finally, we shall highlight important parallels between the two cases and emphasize the universal role of classicality for unitarization.

\section{Classicalization of Goldstones}

We shall adopt the following useful definition of the classical radius $r_{*}$ [2], which is applicable to essentially any interacting theory, regardless of classicalization. With this definition, $r_{*}$ is a classical distance down to which in a high-energy scattering process the wave-packets propagate essentially freely, without experiencing a significant corrections from interaction terms, and beyond which the scattering can no longer be ignored. Simply speaking, $r_{*}$ can be viewed as a distance that defines interaction range at given energy $\sqrt{s}$. Therefore, $r_{*}$ 
automatically determines the cross section as a geometric cross-section

$$
\sigma \sim r_{*}^{2}
$$

With this definition $r_{*}$ is a classical length that survives in the limit $\hbar=0$. The above definition of $r_{*}$ is possible essentially in any interacting theory. The defining property of classicalization is the behavior of $r_{*}$ as a function of energy, relative to other quantum length-scales in the problem. The key property for classicalizing theories is, that for $\sqrt{s} \gg$ $M_{*}, r_{*}$ exceeds all the other quantum length scales in the problem. In particular, $r_{*}(s) \gg$ $L_{*}$ for $\sqrt{s} \gg L_{*}^{-1}$. In such a system the scattering takes place way before the system has any chance of probing distance $L_{*}$.

Following [2], we shall now review the classicalization of scattered wave-packets on a simple prototype example of a derivatively-coupled Nambu-Goldstone type scalar with the following Lagrangian,

$$
\mathcal{L}=\frac{1}{2}\left(\partial_{\mu} \phi\right)^{2}+\frac{L_{*}^{4}}{4}\left(\left(\partial_{\mu} \phi\right)^{2}\right)^{2}
$$

In particular, the above Lagrangian can be viewed as a simplest interacting truncation of Dirac-Born-Infeld-type theory, which is fully sufficient for our purposes. Our results can be easily generalized to higher order non-linearities (see below). This theory is symmetric under the shift by an arbitrary constant $c$,

$$
\phi \rightarrow \phi+c .
$$

The equation of motion is,

$$
\partial^{\mu}\left(\partial_{\mu} \phi\left(1+L_{*}^{4}\left(\partial_{\nu} \phi\right)^{2}\right)\right)=0
$$

In order to identify the $r_{*}$ radius in the above theory, let us consider a scattering process in which for $r=\infty$ and $t=-\infty, \phi$ is well-approximated by a spherical wave of very high center of mass energy $\sqrt{s}=A^{2} / a \gg M_{*} \equiv L_{*}^{-1}$ (where $a$ is a characteristic wave-length) and amplitude $A$,

$$
\phi_{0}=\frac{\psi(r+t)}{r}
$$

which satisfies the free-field equation of motion,

$$
\square \phi_{0}=0 .
$$

Since the initial wave has to describe few quantum particles, we shall assume $A \sim 1$ (small occupation number). We shall now solve the equation (2.4) iteratively, by representing $\phi$-field as superposition of a free wave-packet $\phi_{0}$ and a scattered wave $\phi_{1}$,

$$
\phi=\phi_{0}+\phi_{1} .
$$

We shall treat $\phi_{1}$ as a small perturbation and shall try to understand at what distances this correction to a free wave becomes significant. The classicalization radius will be set by a classical distance at which the approximation

$$
\phi_{1} \ll \phi_{0},
$$

breaks down. 
The equation for the leading correction to the free wave now becomes,

$$
\square \phi_{1}=-L_{*}^{4} \partial^{\mu}\left(\partial_{\mu} \phi_{0}\left(\partial_{\nu} \phi_{0}\right)^{2}\right) .
$$

Taking into the account properties of $\psi(t+r)$-wave, for $a \ll r$, the leading contribution to the right hand side is,

$$
\square \phi_{1}=-\frac{L_{*}^{4}}{r^{5}}\left(2 \psi^{2} \psi^{\prime \prime}+8 \psi \psi^{\prime 2}\right),
$$

where prime denotes the derivative with respect to the argument. For $a^{-1} \gg r^{-1}$ the solution of this equation can be approximated by,

$$
\phi_{1} \simeq-f(r+t) \frac{L_{*}^{4}}{6 r^{4}}
$$

where,

$$
f(r+t) \equiv \int_{0}^{r+t}\left(2 \psi^{2}(y) \psi^{\prime \prime}(y)+8 \psi(y) \psi^{\prime 2}(y)\right) d y .
$$

Notice, that since $\psi$ is a wave-packet of amplitude $\sim 1$ and wave-length $a$, we have,

$$
f \sim \frac{1}{a} \psi .
$$

Thus, the breakdown of the condition (2.8), which signals that scattering became significant, takes place at a distance,

$$
r_{*} \equiv L_{*}\left(L_{*} / a\right)^{\frac{1}{3}} .
$$

Or, translating $a$ in terms of center of mass energy, we can write,

$$
r_{*} \sim L_{*}\left(L_{*} \sqrt{s}\right)^{\frac{1}{3}} .
$$

For example, for a Gaussian wave-packet,

$$
\phi_{0}=A \frac{e^{-\frac{(r+t)^{2}}{a^{2}}}}{r},
$$

the equation (2.10) can be solved exactly in the limit $a \rightarrow 0$,

$$
\phi_{1}(r \gg a)=-\frac{32}{9} \sqrt{\frac{\pi}{3}} L_{*}^{4} A^{3} \frac{\theta(r+t)}{a(t-r)^{3} r},
$$

which confirms (2.14).

Notice, that the physical meaning of the classicalization radius can be understood also in the following way. Consider a probe source $J_{\mu_{1} \mu_{2} \ldots \mu_{3}}$ coupled to $\phi$. Due to the shift symmetry this coupling has to involve gradients of $\phi$. For example,

$$
\left(L_{*}^{2 n} \partial_{\mu_{1}} \phi \partial_{\mu_{2}} \phi \ldots \partial_{\mu_{n}} \phi\right) J^{\mu_{1} \mu_{2} \ldots \mu_{n}} .
$$

In other words, the probe sees an effective background "metric" (potential),

$$
V=\left(L_{*}^{2 n}\left(\partial_{\mu_{1}} \phi\right)\left(\partial_{\mu_{2}} \phi\right) \ldots\left(\partial_{\mu_{n}} \phi\right)\right),
$$

created by $\phi$, and scatters off it. This background becomes order one at $r_{*}$ for $\phi_{1}$ given by (2.17) and with $a \sim r_{*}$. In other words, a softest wave-packet of a fixed energy (and thus of fixed $r_{*}$ ) would-give an order one potential exactly at the distance $r_{*}$. 


\section{Classicalization by gravitons}

We have seen above how a scattering of Goldstone-type scalar wave-packets is classicalized due to self-sourcing. We now wish to consider a situation in which the classicalization of the same wave-packets happens through ordinary gravity.

It is well known that a collapse of a spherical source leads to the formation of a black hole. This is well-known for the sources that are classical to start with. That is, when the occupation number of the initial wave-packet is so large that it can be treated as a classical object. What we wish to see instead is, that exactly the same process for a small initial occupation number can be viewed as classicalization, with classicalization radius $r_{*}$ being equal to the Schwarzschild radius $\left(r_{g}\right)$ corresponding to the center of mass energy of the wave-packet. In other words, classicalization is a precursor effect in which initiallyquantum wave-packet evolves into a configuration with a classical gravitational field that sets an effective range of the interaction.

We consider Einstein's graviton $h_{\mu \nu}$ and a massless Goldstone-type scalar $\phi$. At the level of the free fields, the Lagrangian is,

$$
L_{\text {free }}=-\frac{1}{2} h^{\mu \nu} \mathcal{E}_{\mu \nu}^{\alpha \beta} h_{\alpha \beta}+\frac{1}{2}\left(\partial_{\mu} \phi\right)^{2} .
$$

where

$$
\mathcal{E}_{\mu \nu}^{\alpha \beta} h_{\alpha \beta}=\square h_{\mu \nu}-\eta_{\mu \nu} \square h-\partial_{\mu} \partial^{\alpha} h_{\alpha \nu}-\partial_{\nu} \partial^{\alpha} h_{\alpha \mu}+\eta_{\mu \nu} \partial^{\alpha} \partial^{\beta} h_{\alpha \beta}+\partial_{\mu} \partial_{\nu} h
$$

is a linearized Einstein tensor. Eq. (3.1) represents an unique linear ghost-free action for a massless spin- 2 field. This theory propagates 2 degrees of freedom, and is invariant under the following shift,

$$
\delta h_{\mu \nu}=\partial_{\mu} \xi_{\nu}+\partial_{\nu} \xi_{\mu},
$$

where $\xi_{\mu}$ is an arbitrary vector (below we shall use harmonic gauge $\partial^{\mu} h_{\mu \nu}=\frac{1}{2} \partial_{\nu} h$ ). In order to see how graviton classicalizes in the scattering process, we shall introduce the interaction terms. For our purposes we shall limit ourselves by cubic order interactions in fields. To this order the coupling can be accounted by coupling graviton to the energy momentum tensor of the system. That is, we supplement (3.1) by,

$$
L_{\text {interaction }}=L_{P} h_{\mu \nu} T^{\mu \nu}(\phi)+L_{P} \mathcal{O}\left(h^{3}\right)_{\text {Einstein }}
$$

where, $L_{P} \equiv M_{P}$ is the Planck length, and

$$
T_{\mu \nu}(\phi)=\partial_{\mu} \phi \partial_{\nu} \phi-\frac{1}{2} \eta_{\mu \nu}\left(\partial_{\alpha} \phi \partial^{\alpha} \phi\right)
$$

and $\mathcal{O}\left(h^{3}\right)_{\text {Einstein }}$, stands for a cubic part of the Einstein's action, which can be thought of as a self-sourcing of graviton by its own energy-momentum tensor. For simplicity, we shall not write this term explicitly in the action, but only its contribution into the equation of motion. To this order (and using harmonic gauge) the equation of motion for the graviton has the following form,

$$
\square h_{\mu \nu}=-L_{P}\left(T_{\mu \nu}-\frac{1}{2} \eta_{\mu \nu} T_{\alpha}^{\alpha}\right)
$$


where

$$
T_{\mu \nu} \equiv T_{\mu \nu}(\phi)+T_{\mu \nu}(h)
$$

and

$$
\begin{aligned}
T_{\mu \nu}(h)= & -\frac{1}{2} h^{\alpha \beta}\left(\partial_{\mu} \partial_{\nu} h_{\alpha \beta}+\partial_{\alpha} \partial_{\beta} h_{\mu \nu}-\partial_{\alpha}\left(\partial_{\nu} h_{\mu \beta}+\partial_{\mu} h_{\nu \beta}\right)\right)- \\
& -\frac{1}{2} \partial_{\alpha} h_{\beta \nu} \partial^{\alpha} h_{\mu}^{\beta}+\frac{1}{2} \partial_{\alpha} h_{\beta \nu} \partial^{\beta} h_{\mu}^{\alpha}-\frac{1}{4} \partial_{\mu} h_{\alpha \beta} \partial_{\nu} h^{\alpha \beta} \\
& \left.-\frac{1}{4} \eta_{\mu \nu}\left(\partial_{\alpha} h_{\beta \gamma} \partial^{\beta} h^{\alpha \gamma}-\frac{3}{2} \partial_{\alpha} h_{\beta \gamma} \partial^{\alpha} h^{\beta \gamma}\right)\right) \\
& -\frac{1}{4} h_{\mu \nu} \square h+\frac{1}{2} \eta_{\mu \nu} h_{\alpha \beta} \square h^{\alpha \beta} .
\end{aligned}
$$

Since, we have already discussed the classicalization due to self-sourcing of $\phi$, we shall not include the self-interaction terms for simplicity, but only terms that source graviton.

We shall now proceed as follows. We assume that our system starts at $t=-\infty$ and $r=\infty$ in an "in"-state with no gravitons and a collapsing free wave-packet of $\phi=\phi_{0}$ with a trans-Planckian center of mass energy and a small occupation number.

Perturbatively, such a $\phi$-scattering due to graviton exchange would violate unitarity for $\sqrt{s} \gg M_{P}$. Instead we shall see that system classicalizes via production of a large gravitational field.

We shall observe how the initial quantum wave will evolve into a classical configuration of the graviton field, and how the classicalization distance will depend on energy. In order to achieve this goal, we shall look for the solution for graviton in form of an expansion,

$$
h_{\mu \nu}=h_{\mu \nu}^{(0)}+h_{\mu \nu}^{(1)}
$$

where $h_{\mu \nu}^{(0)}$ is the solution of the linear equation, that takes into the account sourcing of graviton by $T_{\mu \nu}(\phi)$ (but ignores self-sourcing!), whereas, $h_{\mu \nu}^{(1)}$ is the next order correction due to self-sourcing by $T_{\mu \nu}(h)$. We than try to find out at what radius,

$$
h_{\mu \nu}^{(0)} \sim M_{P}
$$

and

$$
h_{\mu \nu}^{(1)} \sim h_{\mu \nu}^{(0)} .
$$

This will define the classicalization radius, $r_{*}$. We shall discover that this distance is set by the classical scale equal to a Schwarzschild radius corresponding to a center of mass energy. This is a clear signal that system classicalizes by formation of classical objects whose size is governed by the Schwarzschild radius. Let us now analyze the scattering process. We choose, $\phi_{0}$ to be a free spherical wave-packet,

$$
\phi_{0}=\frac{\psi(r+t)}{r},
$$

of amplitude $A$ and energy $\sqrt{s} \equiv M \sim A^{2} / a$, where $1 / a$ is a characteristic momentum (or inverse localization width) entering in the wave-packet. For evaluating $h_{\mu \nu}^{(0)}$ we need to solve equation (3.6) with the source (3.5), which takes the following form,

$$
\square h_{\mu \nu}^{(0)}=-L_{P}\left(\partial_{\mu} \phi_{0} \partial_{\nu} \phi_{0}\right) \text {. }
$$


Let us evaluate this equation on a spherical wave-packet. Picking up (a most interesting) $h_{00}$ component of the graviton that in classicalized limit should reproduce Newtonian potential of a classical source, we get

$$
\square h_{00}^{(0)}=-L_{P} \frac{\psi^{\prime 2}}{r^{2}},
$$

where prime denotes derivative with respect to the argument (in this case $(t+r)$ ). Is is obvious that for any localized wave-packet with total center of mass energy $M$, outside the source the Newtonian gravitational potential must behave as $h_{00}^{(0)} \sim L_{P} M / r$, which makes it clear that the classicalization radius is given by the Schwarzschild radius associated with the center of mass energy $M$.

To make the analogy with the Goldstone case deeper, we wish to represent the metric in the form that is most convenient for confronting it with the case of a classicalized scalar field (2.17). For this, let us take

$$
\phi_{0}=\frac{\psi(r+t)}{r}=A \frac{e^{-\frac{(r+t)^{2}}{2 a^{2}}}}{\pi^{1 / 4} r},
$$

which represents a wave-packet of amplitude $A$ and energy $M \sim A^{2} / a$. The equation (3.14) now becomes (irrelevant factors are absorbed in $L_{P}$ ),

$$
\square h_{00}^{(0)}=-2 L_{P} M \frac{1}{r^{2}}\left(1+\frac{1}{2} a^{2} \partial_{t}^{2}\right) \frac{e^{-\frac{(r+t)^{2}}{a^{2}}}}{a \sqrt{\pi}}
$$

For clarity of the solution, we shall take a limit $a \rightarrow 0, A^{2} / a=$ fixed, and use the relation

$$
\lim _{a \rightarrow 0} \frac{1}{a \sqrt{\pi}} e^{-\frac{(r+t)^{2}}{2 a^{2}}}=\delta(r+t) .
$$

The equation then becomes,

$$
\square h_{00}^{(0)}=-2 L_{P} \frac{M}{r^{2}} \delta(r+t)
$$

which gives,

$$
h_{00}^{(0)}=\frac{L_{P} M}{r} \theta(r+t) \ln (r-t) .
$$

Comparison of this expression with (2.17) suggests a clear analogy between the two cases. In particular, it is obvious that in gravity $L_{P}$ plays the role of $L_{*}$, whereas the role of $r_{*}$ is played by the Schwarzschild radius associated with energy $\sqrt{s} \equiv M$. Indeed, classicalization radius is set by the condition (3.10), which is reached at $r_{*} \sim L_{P}^{2} M$, and which is nothing but the Schwarzschild radius of a black hole of mass $M$.

Note, that appearance of a time-dependent log-factor in (3.19), is an artifact of the harmonic gauge we are working in. In this gauge, appearance of the log-factors in the gravitational tails at $1 / r^{2}$-order in source expansion is a well known feature in gravitational wave physics, and can be removed by appropriate gauge shift, which effectively amounts to a correction of light-cone outside the source [24-27]. However, for our purposes, of 
making the parallel between the graviton and Goldstone classicalization more transparent, we prefer to stay in the above gauge.

Now evaluating (3.8) on (3.19) and inserting it eq. (3.6) we can get the standard corrections to the metric at second order in $L_{P}^{2}$. For example,

$$
\frac{h_{00}^{(1)}}{M_{P}} \sim \frac{1}{2} \frac{r_{*}^{2}}{r^{2}}
$$

which clearly indicates that the second condition of classicalization (3.11) is also met at $r \sim$ $r_{*}$. We thus see, that the classicalization radius for spin-2 coincides with the Schwarzschild radius corresponding to the center of mass energy. For the interested reader, there is a full non-linear treatment for the collapse of a spherical light shell confirming our findings for the formation of the classicalon (black hole in this case) [28], for us however most important is the deconstruction of the collapse from classicalization point of view, as we did above.

\section{Classicalization of graviton scattering}

We shall now study the classicalization of graviton scattering. For this we shall repeat the same exercise as in the previous case, but replace an incoming scalar wave-packet by a graviton one. We shall thus again solve the equation (3.6) iteratively, by setting the scalar field to zero, and taking $h_{\mu \nu}^{(0)}$ as a free incoming gravitational wave-packet. All the machinery required for such analysis has already been prepared in series of excellent papers [24-27, 29] studying both the multipole and the post-Newtonian expansions of gravitational waves. We shall limit ourselves by adapting the key essentials of this analysis for our purpose of understanding classicalization of scattered gravitons.

For standard convenience we rewrite the equation (3.6), in notation of trace-reversed graviton,

$$
\bar{h}_{\alpha \beta}=h_{\alpha \beta}-\frac{1}{2} \eta_{\alpha \beta} h_{\kappa}^{\kappa},
$$

which in harmonic gauge $\partial_{\mu} \bar{h}^{\mu \nu}=0$ takes the form,

$$
\square \bar{h}^{\mu \nu}=-\frac{1}{M_{P}} \Lambda^{\mu \nu}
$$

where $\Lambda^{\mu \nu}$ is given by

$$
\begin{aligned}
\Lambda_{\mu \nu}(\bar{h})= & -\bar{h}^{\kappa \lambda} \partial_{\mu} \partial_{\nu} \bar{h}_{\kappa \lambda}+\partial_{\kappa} \bar{h}^{\mu \lambda} \partial_{\lambda} \bar{h}^{\nu \kappa}+\frac{1}{2} \eta^{\mu \nu} \partial_{\lambda} h_{\kappa}^{\sigma} \partial_{\sigma} \bar{h}^{\kappa \lambda} \\
& -\partial_{\lambda} \bar{h}^{\nu \tau} \partial^{\mu} \bar{h}_{\tau}^{\lambda}-\partial_{\lambda} \bar{h}^{\mu \tau} \partial^{\nu} \bar{h}_{\tau}^{\lambda}+\partial_{\lambda} \bar{h}^{\mu \tau} \partial^{\lambda} h_{\tau}^{\nu} \\
& +\frac{1}{8}\left(2 \eta^{\mu \lambda} \eta^{\nu \kappa}-\eta^{\mu \nu} \eta^{\kappa \lambda}\right)\left(2 \partial_{\kappa} \bar{h}^{\tau \sigma} \partial_{\lambda} \bar{h}_{\tau \sigma}-\partial_{\kappa} \bar{h}_{\tau}^{\tau} \partial_{\lambda} \bar{h}_{\sigma}^{\sigma}\right)
\end{aligned}
$$

We shall solve this equation iteratively by representing a graviton as a superposition of free and scattered waves,

$$
h_{\mu \nu}=h_{\mu \nu}^{(0)}+h_{\mu \nu}^{(1)},
$$

which to the leading order satisfy the following equations,

$$
\square \bar{h}_{\mu \nu}^{(0)}=0, \quad \partial_{\mu} \bar{h}_{\mu \nu}^{(0)}=0
$$


and

$$
\square \bar{h}_{\mu \nu}^{(1)}=-\frac{1}{M_{P}} \Lambda^{\mu \nu}\left(h^{(0)}\right)
$$

The solution to (4.5) is usually given in terms of infinite multipolar series using symmetric trace-free harmonics [29] and takes the form of multipolar waves

$$
\bar{h}^{(0) \mu \nu}=\sum_{\ell=0}^{\infty} \partial_{L}\left(\frac{K_{L}^{\mu \nu}(u)}{r}\right)
$$

Here $L=i_{1} i_{2} \ldots i_{\ell}$ is a compact notation for multipolar index of $\ell$ spatial indices. Thus, $K_{L}^{\mu \nu}=K_{i_{1} i_{2} \ldots i_{\ell}}^{\mu \nu}$ and $\partial_{L}=\partial_{i_{1}} \partial_{i_{2}} \ldots \partial_{i_{\ell}}$. In particular $K_{i_{1} i_{2} \ldots i_{\ell}}^{\mu \nu}$ in our case is a function of $u \equiv t+r$ and it is symmetric and trace-free in its lower indices.

For simplicity we shall choose incoming wave to be a quadrupole,

$$
\begin{aligned}
& \bar{h}_{00}^{(0)}=-2 \partial_{i} \partial_{l}\left(\frac{M_{i l}(u)}{M_{P} r}\right), \\
& \bar{h}_{0 i}^{(0)}=2 \partial_{j}\left(\frac{M_{j i}^{(1)}(u)}{M_{P} r}\right), \\
& \bar{h}_{i j}^{(0)}=-2 \frac{M_{i j}^{(2)}(u)}{M_{P} r},
\end{aligned}
$$

where $M_{k l}^{(n)}=d^{n} M_{k l} / d t^{n}$.

The fact, that very similar classicalization mechanism is at work as in the case of scalar scattering, can already be anticipated from the observation, that when evaluated on (4.9) to the leading order of $1 / r^{2}$ contribution, the right hand side on the equation (4.6) takes the form of the stress energy tensor of a massless field,

$$
\square \bar{h}_{\mu \nu}^{(1)}=-\frac{1}{M_{P}} \frac{1}{r^{2}} k_{\mu} k_{\nu} \Pi(t+r)
$$

where, $k_{\mu}$ is null vector, and quantity $\Pi$ accounts for the multipole structure of the product of the two quadrupoles, and can be re-expanded in the standard way.

In order not to stress the reader with a tedious but straightforward calculation of general case (which can be found in standard gravitational wave analysis [24-27, 29]), we limit ourselves by illustrating the point for a monopole component of $\Pi(t+r)$, in which case the problem essentially reduces to a scalar incoming wave studied in the previous section. So the gravitational $h_{\mu \nu}^{(1)}$ field produced by a monopole component of the self-source is now of the same nature as the field $h_{\mu \nu}^{(0)}$ produced by sourcing by a scalar wave in the equation (3.19). For a sharply localized incoming free wave-packet, the $h_{00}^{(1)}$ produced by a monopole component of a self-source can be approximated by

$$
h_{00}^{(1)} \sim \frac{L_{P} \sqrt{s}}{r} \theta(t+r) \ln (r-t),
$$

where $\sqrt{s}$ is (a spherically averaged) center of mass energy of an incoming wave. Just as in the scalar case, this fixes the classicalization radius at the Schwarzschild radius corresponding to this center of mass energy $r_{*} \sim r_{g}=\sqrt{s} L_{P}^{2}$. 


\section{$5 \quad$ Parallels and differences}

\subsection{Classicalons in gravity versus Goldstone}

We have proven the emergence of the classical radius $r_{*}$ in the scattering process, both for gravitons as well as Goldstones. In both cases this classical radius marks an effective range of interaction, that is, a distance where a free-wave approximation breaks down. In both cases a correction to a free wave is leaving behind a power-law tale. All the above signals, that we deal with a configuration that became classical at $r_{*}$. Thus, whatever the subsequent evolution of the system is, an $s$-wave scattering of highly energetic quanta leads to a formation of a classical field configuration of size $r_{*}$. In neither cases this classicalon configuration yet corresponds to any static spherically-symmetric solution.

What is a post-classicalization evolution of fields?

In case of gravity the answer is simple. Classicalization must be a precursor of the formation of a Schwarzschild black hole. This is obvious from the spherical symmetry of the problem and from Birkhoff's theorem. Of course, the details of horizon crossing cannot be captured by our analysis, but there is no need for it. Our goal was to witness classicalization.

What is an analogous static solution (if any) in the scalar case to which the classicalon configuration may settle? As found in [1], theory does admit such static singular solutions, which in some respect are analogous to black holes. Whether a time-dependent classicalon configuration will settle to this, is unclear. But, the key point is, that from the unitarization point of view there is no need for a settlement to any stable or long-lived solution, since classical configuration has already being formed and this is enough. Once formed, the classical configuration cannot decay in a two-particle state without paying price of exponentially-suppressed probability.

This simple but important fact, naturally allows us to resolve the question of the role of black hole-type entropy for unitarization of the scattering amplitude. Since black hole entropy is a property of very special classical configurations, whereas any intermediate classicalon that is formed by self-sourcing suffices to unitarize the scattering process, it follows that black hole entropy is just an accompanying property for gravitational systems (which of course automatically agrees with the counting of exponential suppression in twoparticle decays) but not a defining property for unitarization.

\subsection{The role of entropy in unitarization by classicalization}

The closed parallel presented here between gravity and Goldstone examples, clarifies a potential misconception about the role of black hole entropy in unitarization process. We wish to discuss this point in more details. The process of unitarization by classicalization consists of two ingredients.

(a) First is to show, that an initial two-particle state inevitably leads to the development of classical configuration. We have demonstrated this for an $s$-wave scattering.

(b) The second ingredient is the fate of this classical configuration: An exponential suppression of its decay-probability into a two-particle state. 
Let us investigate the role of black-hole-type entropy for any of these stages. Obviously, entropy plays no role at the first (classicalization) stage neither in Goldstone nor in gravity examples. We saw, that classicalization took place before one could talk about any entropy or even a well-defined horizon.

What role does entropy play in the second aspect? In gravity example, entropy is a byproduct of classicalization, since we know that a spherical wave packet after collapsing will form a Schwarzschild black hole with an associated Bekenstein-Hawking entropy. So in gravity, at least in the $s$-wave scattering, the outcome of classicalization is a black hole formation, and this is accompanied by formation of entropy. However, main reason for unitarization is classicality, not the entropy of the final classical configuration. Any classical configuration, with or without entropy, can only decay into a two-particle state with exponentially-suppressed probability. Since entropy is a inevitable accompanying property of classicalons in gravity case, entropy counting does agree with this suppression. But classicality is the key reason for the suppression regardless of entropy. For example, a binary system of neutron and anti-neutron stars carries no black hole type entropy, but probability for it to decay into two photons is obviously suppressed.

This is a defining property of all classical configurations. They represent many particles in coherent state and due to this their decay into any two-particle states is exponentially suppressed, by a factor $e^{-N c}$, where $N$ is number of soft quanta composing the configuration in question, $c$ is a factor of order one up to $\log N$ corrections. An exact computation of this suppression factor is beyond the scope of our paper, but it is very instructive to estimate it from the following reasoning.

The classicalon configuration of size $r_{*}$ and mass $\sqrt{s}$ is mostly composed out of soft quanta of wave-length $\sim r_{*}$. Therefore their number in a classicalon configuration can be estimated as $N \sim \sqrt{s} r_{*}$, and thus the decay into two particles is suppressed by a factor,

$$
\Gamma_{\text {classicalon } \rightarrow \text { few }} \sim \mathrm{e}^{-\sqrt{s} r_{*}} .
$$

Notice that for the gravity case $\left(r_{*}=r_{g}=\sqrt{s} L_{P}^{2}\right)$ the above expression correctly reproduces entropy suppression of a black hole decay into few particles, although we never referred to any entropy!

\subsection{If not $N \rightarrow 2$, then why $2 \rightarrow N$ ?}

We now wish to address a question that may bother some readers.

We have just argued that two-particle decay of any classical configuration, which can always be understood as a superposition of many quanta, is exponentially suppressed. In this light how can we understand an unsuppressed formation of the same classical state out of initial few energetic quanta?

One may think that in the case of a black hole the large entropy factor somehow plays the role and overcompensates. This way of counting is misleading. First, as we know, the gravitational field classicalizes before horizon is even formed and one can prescribe standard Bekenstein-Hawking entropy. The two-body decay of such a classical field would be suppressed even if it never evolves into a black hole. Moreover, the origin of the black 
hole's entropy is precisely the result of the fact that many initial states evolve into the same final one, and not other way round.

The fact that the same black hole can be formed by many different initial states, gives no enhancement for black hole formation from each initial pure state.

So the answer to the above question has nothing to do with the particularities of the black hole entropy, but rather again is universal property of classicallization. To answer this question let us notice, that when we form a classical configuration out of an initial wave-packet describing free particles, we are dealing with a transition that takes infinite time, because initial particles can be considered as free only if $r \gg r_{*}$.

So the $N$-particle classical configuration is built up gradually, during a very long time, and at distances at which the quantum re-scattering is negligible. Of course, there is full time reversal symmetry, and $N$-particle state could gradually bounce back into the original 2-particle state, however, the time needed for this is much longer than the time of rescattering among the $N$-particles, which takes away the needed coherence. As a result, smooth bounce back almost never happens.

Also, since classicalization (both for gravity and other cases) forces the system to produce $N$ soft quanta, the enhancement can only be encoded in the number of states in which these particles can be produced, and must be universally applicable for gravity and for other cases.

Let us analyze this discussion in an universal field theoretic language, equally applicable both for black holes and as well as for other classicalons. Consider an above-considered scattering process in a theory (not necessarily a classicalizing one) in which the interaction range $r_{*}(s)$ is some function of center of mass energy $\sqrt{s}$. This means that highly energetic wave-packet starts to scatter at distance $r_{*}$. Typical quanta produced in this scattering have wave-length $r_{*}(s)$. Their available number can be estimated as $N=\sqrt{s} r_{*}(s)$, for $r_{*}(s) \geq 1 / \sqrt{s}$ and $N \sim 1$ in the opposite case $r_{*} \leq 1 / \sqrt{s}$. . Let us ask, what is the suppression factor for producing a composite object of the available mass $\sqrt{s}$ composed out of quanta of wave-length $\bar{r}$. The number of quanta in such an object will of course be $\bar{N} \equiv \sqrt{s} \bar{r}$. Up to log-corrections in the exponent, the exponential suppression factor for such a process will be

$$
\mathrm{e}^{-\frac{\bar{r}}{r_{*}(s)}} \equiv \mathrm{e}^{-\frac{\bar{N}}{N}}
$$

Notice that this universal language correctly reproduces the suppression price for soliton production in high energy scattering in weakly coupled (non-classicalizing) theories, because in such theories at high energies $r_{*}(s) \ll 1 / \sqrt{s}$, whereas, because size of the soliton $\bar{r}$ is much larger than its inverse mass $\bar{r} \gg 1 / \sqrt{s}$, hence an exponentially suppressed price. For example, for t'Hooft-Polyakov monopoles, in a weakly coupled theory with gauge-coupling $g$, the monopole size and mass in terms of gauge boson mass are given by $1 / m_{W}$ and $m_{W} / g^{2}$ respectively. The minimal center of mass energy for pair production is $\sqrt{s} \sim m_{W} / g^{2}$. Thus, we have $\bar{r} \sim\left(1 /\left(\sqrt{s} g^{2}\right)\right)$, whereas $r_{*}(s) \sim g^{2} / \sqrt{s}$. Hence for a production of monopole anti-monopole pair in scattering of two particle we obtain the following suppression,

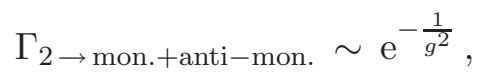


which very well reproduces the expected suppression for production of solitonic objects. However, when applied to classicalons the same method shows that suppression disappears. This is because, for classicalizing theories $r_{*}(s) \gg 1 / \sqrt{s}$ and $\bar{r}=r_{*}(s)$. So the exponent becomes one! This explains why production of black holes is unsuppressed, without ever referring to any entropy.

The physics of suppression is entirely clear in terms of soft quanta. The rule is simple. Suppression in a production of a classical object of size $\bar{r}$ appears whenever the number of soft quanta required for making up this object $(\bar{N})$ is larger than the number of soft quanta $N$ that the system can produce at the distance $r_{*}(s)$. For weakly coupled solitons $\bar{N} \gg N$, and the suppression is given by (5.3).

For black holes and other classicalons, these two numbers are similar, $\bar{N} \simeq N$ and the suppression disappears. In particular case for black holes, $r_{*}(s)$ is the Schwarzschild radius and $N \sim r_{g} \sqrt{s}$, which is the same as entropy, but the physics is much more general, and what matters is simply the number of soft quanta produced by scattering.

Having understood in this language why classicalon formation avoids any price of exponential suppression that one would naively expect, let us now understand the suppression of the two particle decay in the same language. This suppression is simply coming from the fact that $N$ soft quanta that compose the classicalon have to annihilate into 2 very energetic ones. This obviously costs (5.1), which for the black hole automatically reproduces the entropy or Boltzmann suppression, but there is no need to refer to any of these notions. As we see the answer can be understood in very general terms of need to annihilate many soft quanta into the two energetic ones.

There no violation of time reversal symmetry or any other exotica in physics of classicalons. Rather the physics is simple. Production of classicalon in high energy collision is easy because in classicalizing it is easy to produce many soft quanta out of enough energy. However, once soft quanta have been produces their annihilation into few energetic ones is always costly.

\section{Classicalization landscape: gravity as a most efficient classicalizer}

\subsection{Graviton as a most efficient classicalizer}

In our treatment of the scalar example, the effect of classicalization on the scattering amplitude can also be represented in the following terms. We have started with a theory in which at low energies $\left(\sqrt{s} \ll L_{*}^{-1}\right)$ an $s$-channel $2 \rightarrow 2$ scattering amplitude behaves as $[8,10]$,

$$
\left.A(s)\right|_{s \ll L_{*}^{-1}} \sim \frac{L_{*}^{2} s^{2}}{t} .
$$

Naive extrapolation of the above perturbative amplitude at high energies would imply violation of unitarity for $\sqrt{s} \gg L_{*}^{-1}$. Instead, the amplitude behaves as,

$$
\left.A(s)\right|_{s \gg L_{*}^{-1}} \sim\left(\frac{L_{*}}{r_{*}(s)}\right)^{2},
$$


with $r_{*}(s)$ being given by (2.15). This is the key idea of unitarization by classicalization. Since, the high energy scattering takes place at the classical radius $r_{*}$, two-to-two scatterings are dominates by momentum-transfer of order $r_{*}(s)^{-1}$. In a generic classicalizing theory the $\sqrt{s}$-dependence of $r_{*}$ can be parameterized as,

$$
r_{*}(s)=L_{*}\left(\sqrt{s} L_{*}\right)^{\alpha},
$$

where for classicalization it is necessary that $\alpha$ is a positive number. Its precise value depends on the self-sourcing interaction. As we have seen, for a graviton $\alpha=1$.

The physical meaning of (6.2) can be easily understood as a consequence of the transformation of a hard quanta of energy $E$ into a set of $N(E)$ soft quanta of wave length $\frac{1}{r_{*}(E)}$ induced by energy self sourcing. In the particular case of gravity $N(E)=s L_{P}^{2}=E r_{*}(E)$. In the case we are considering of a $2 \rightarrow 2$ scattering process among gravitons with total energy $s$, the average number of elementary interaction vertices contributing to the process is $N(s)$. This directly follows from the fact that the interaction among gravitons is set by $L_{P}^{2}$. In the limit of $s \gg t$ the main contribution comes from exchange of transfer momentum in the $t$ channel. For $t \gg M_{P}^{2}$ the Born approximation to this process will violates unitarity. However we should expect, as the leading contribution, those processes where the total $t$ is exchanged through $N(s)$ elementary exchanges $[11,12,15,18,19]$. This leads to an effective momentum transfer $\frac{t}{N(s)} \sim \frac{1}{r_{*}(s)}$ and to expression (6.2). More concretely in theories that classicalize we can define $N(E)=E r_{*}(E)$ and unitarization proceeds by replacing $s$ and $t$ by $\frac{s}{N^{2}(s)}$ and $\frac{t}{N^{2}(t)}$ respectively.

At this point the reader can wonder how this form of softening amplitudes can be consistent with black hole formation in those kinematical regimes where we have for a given $s$, an impact parameter smaller than the corresponding gravitational radius. Here, is important to disentangle the classical analysis of black hole formation as the appearance -in certain kinematical initial conditions- of trapped surfaces and the pure $S$ matrix approach to the problem. The softening of the amplitude in the $s$ channel is due to transforming the energy $s$ carried by the $\mid$ in $>$ hard quanta into a set of $N(s)$ soft quanta localized in a region of typical size $r_{*}(s)$. Black hole ( or classicalon) formation depends on the very non perturbative issue of when these localized soft quanta in the $s$ (or $t$ ) channel become a new resonance state in the spectrum contributing to the imaginary part of the amplitude. Classicality approaches this question observing that the set of localized $N$ soft quanta define a classical state in the sense that all the dynamical scales involved are bigger than the Compton lengths. This classical state contributes to the $2 \rightarrow 2$ amplitude as $e^{-N}$. It is this extra exponential suppression the sign -in $S$ matrix language- of black hole (or classicalon) formation.

Clearly we are not claiming that classicalization is the only way to unitarize scattering amplitudes. Our point is that classicalization is most important for scattering at small impact parameters (smaller than $r_{*}$ ). Let us deconstract this statement. Consider scattering of two quanta (say gravitons or $\phi$-fields) by gravitational interaction, at some large $\sqrt{s}$. At large impact parameter $\left(\gg r_{*}\right)$ the seeming violations of unitarity are resolved by eikonal resummation $[8]-[18,19]$ and we have nothing more to add to this knowledge. Classicalization becomes important for violating unitarity at small impact parameter $\left(\ll r_{*}\right)$, because 
as we have shown in non-perturbative treatment (on an example of s-wave scattering) the small impact parameter scattering results into formation of a classical configuration, and thus automatic suppression of $2 \rightarrow 2$ scattering. So at small impact parameter, the $2 \rightarrow 2$ scattering can only take place in two ways: 1 ) particles scatter with high momentum transfer, form a classical configuration which then decays back to two particle state. This amplitude is exponentially suppressed. 2) Second option is that particles scatter before coming closer than their $r_{*}$ radius. In such a case, momentum transfer is very low, $\lesssim 1 / r_{*}$, and on dimensional grounds the amplitude goes as eq. (6.2).

Let us now show, that in Poincare-invariant theories with a scalar classicalizer field, $\alpha$ has to be below one. Following [1], consider a (self)sourcing by an operator that contains $2 k$ power of derivatives and $n$ power of a scalar field $\phi$, which schematically can be written as

$$
G_{\phi} \partial^{2 k} \phi^{n}
$$

where $G_{\phi}$ is a coupling constant of dimensionality $G_{\phi}=[\text { mass }]^{\frac{2-n}{2}}[\text { length }]^{\frac{n+4 k-6}{2}}$. This coupling defines a quantum length-scale $L_{*}=G_{\phi}^{\frac{1}{n+2 k-4}} \hbar^{\frac{n-2}{(n+2 k-4)}}$ that marks the breakdown of perturbative unitarity.

The classicalization radius caused by such an operator, can be estimated by noticing that the only classical length that can be constructed out of the scale $L_{*}$ and a de Broglie wave-length of the wave-packet $a=\frac{\hbar}{\sqrt{s}}$ is

$$
r_{*}=L_{*}\left(\frac{L_{*}}{a}\right)^{\frac{n-2}{n+4 k-6}} .
$$

Of course, the above dimensional analysis does not prove that the above operator always leads to classicalization, but whenever it does, the classical radius scale is uniquely defined and since $k \geq 2$ ( $k=2$ corresponds to a free theory by field redefinition), we have $\alpha=$ $\frac{n-2}{n+4 k-6}<1$.

For the purposes of our present discussion, more reliable proof that $\alpha<1$, is to show that the length scale at which the wave-packet first gets perturbed by self-interaction, which therefore must always be equal or larger than $r_{*}$, must scale as fractional power of $1 / a$.

Let us estimate such radius caused by such an operator in the above-considered scattering process. The self-sourcing equation (analog of (2.9)) can be schematically written as,

$$
\square \phi_{1}=L_{*}^{2 k+n-4} \partial^{2 k} \phi_{0}^{n-1},
$$

where $\phi_{0}$ is an initial free wave-packet (given by (2.5)) of characteristic wave-length $a$ and energy $\sqrt{s} \sim 1 / a$. The ordering of derivatives and fields is model-dependent and is not shown explicitly. Because $\phi$ is a scalar, all the Lorentz-indexes of derivatives on the r.h.s. of the equation (6.6) are contracted among each other. Due to this fact, for $a \ll r$ each pair of contacted derivatives results into a factor $1 /(a r)$ as opposed to $1 / a^{2}$ that one would naively anticipate. So in total the effect of derivatives is crudely reduced to an appearance of a factor of order $1 /(r a)^{n}$. Thus generically, the equation will have a form,

$$
\square \phi_{1} \sim L_{*}^{2 k+n-4} \frac{1}{a^{k}} \frac{f(r+t)}{r^{n+k-1}},
$$


where, $f(r+t)$ is a wave-packet of wave-length $a$ and of order-one amplitude. In order to solve for $\phi_{1}$ we have to invert the box, which effectively removes one power of $1 /(a r)$, so that finally we have,

$$
\phi_{1} \sim L_{*}^{2 k+n-4} \frac{1}{a^{k-1}} \frac{\tilde{f}(r+t)}{r^{n+k-2}},
$$

where,

$$
\tilde{f}(r+t) \equiv a \int_{0}^{r+t} f(y) d y .
$$

Since, both $\psi$ and $\tilde{f}$ have order-one amplitudes, the $\hat{r}$ radius, which marks the breakdown of the free-wave approximation $(2.8)$, is given by $\hat{r} \sim L_{*}\left(L_{*} / a\right)^{(k-1) /(n+k-3)}$. Or, translated in terms of center of mass energy of the initial wave-packet, we can write,

$$
\hat{r} \sim L_{*}\left(L_{*} \sqrt{s}\right)^{(k-1) /(n+k-3)} .
$$

The key point is, that by default for any system $\hat{r}$ represents an upper bound on $r_{*}$, since system cannot classicalize before experiencing interaction. Notice, also that for $k=n / 2$, $\hat{r}=r_{*}$.

It may be instructive to see this more transparently by taking a limit of a sharply localized wave-packet, $\phi_{0}=A e^{-\frac{(r+t)^{2}}{a^{2}}}$. Taking now a limit $a \rightarrow 0$ and $\sqrt{s} \sim A^{2} / a=$ fixed, the function $f(r+t)$ in (6.7) becomes $\delta(r+t)$,

$$
\square \phi_{1}=L_{*}^{2 k+n-4} \frac{A^{n-1}}{a^{k-1}} \frac{\delta(r+t)}{r^{n+k-1}},
$$

(here and below irrelevant numerical factors will be dropped). Which can be easily integrated and gives the following expression for $\phi_{1}^{(1)}$,

$$
\phi_{1}=L_{*}^{2 k+n-4} \frac{A^{n-1}}{a^{k-1}} \frac{\theta(r+t)}{(t-r)^{n+k-3} r}=L_{*}^{2 k+n-4} \frac{A^{n-2}}{a^{k-1}} \frac{\theta(r+t)}{(t-r)^{n+k-3}}\left(\frac{A}{r}\right)
$$

In the very last expression, we have singled out a factor $A / r$ for convenience of comparing with $\phi_{0}$ which is of order $A / r$. Taking $2 k=n$ and comparing this with $\phi_{0}$, keeping in mind that $A^{2} / a \sim \sqrt{s}$, we get that $r_{*}=\hat{r}$ given by $(6.10)$.

For $k>n / 2$ we can apply the same counting, but one has to make sure that the background is ghost-free, on case by case basis, and we won't enter in the details here. Some interesting ideas appeared recently about self-protection of such systems [30] as well as about generalized high-derivative candidates for classicalizing theories [31].

For us important thing is that, since, $\hat{r} \geq r_{*}$, confronting with (6.3), we see, that for scalar theories $\alpha \leq(k-1) /(n+k-3)$ and since $n>2$, we have $\alpha<1$.

The reason why spin-2 case avoids this power-counting is clear from the equation (3.6), which is a tensor equation, and derivatives acting on wave-packet on the r.h.s. are not Lorentz-contracted with each other. As a result, the r.h.s. of the equation,

$$
\square h_{00}^{(1)}=-L_{P}\left(T_{00}-\frac{1}{2} T_{\alpha}^{\alpha}\right)
$$


when evaluated on a similar free wave-packet (of energy $\sqrt{s}=1 / a$ and small occupation number), is of order $\sim 1 /(a r)^{2}$, as opposed to $1 / a r^{3}$ that it would be had we replaced graviton with a scalar with a two-derivative cubic interaction. The resulting $r_{*}$ therefore is $r_{*}=L_{*}^{2} \sqrt{s}$.

In other words, we observe, that graviton is a most efficient classicalizer!

\subsection{Generalized holographic bound on information storage}

Given the fact, that classicalizing theories are based on the concept of a minimal length $\left(L_{*}\right)$ and a classical radius $\left(r_{*}(s)\right)$ associated with the energy, they allow for a generalization of the black hole bound on information storage. Because the latter bound involves the area, often it is refereed to as the holographic bound. We shall see, that in a classicalizing theory the analog of this bound can be derived and can be interpreted as the bound on the number of soft quanta $N$ that is required to make up a given classicalon configuration.

Because of this, the landscape of classicalizing theories can also be parameterized according to this bound. As we shall see, not surprisingly, gravity gives a most stringent bound. Of course, in each case, the bound has to be understood as the bound on amount of information that can be stored in the quanta that exhibit classicalization.

In order to derive the bound, let us consider a classicalizing theory with $r_{*}$ given by (6.3). Imagine localizing a bit of information within a region of size $r$. Since information is encoded in particles, such a localization cost at least energy $\sim 1 / r$. Correspondingly, for $N$ bits this energy is at least $\sqrt{s} \sim N / r$. This lower bound on needed energy is independent of how the particles carrying this information interact. In a weakly-coupled non-classicalizing theory, one can store an arbitrary amount of information within a fixed region $r$, by pumping-in a sufficient amount of energy. However, in a classicalizing theory this is not possible, since required energy makes the $r_{*}$ radius of the information-storage grow according to (6.3), and thus, resists localization. The $r_{*}$ corresponding to the abovementioned energy-storage is

$$
r_{*}(N)=L_{*}\left(L_{*} N / r\right)^{\alpha}
$$

A given amount of information ( $N$-bits) can be contained within a volume $r$ as long as $r<r_{*}(N)$. Thus, the maximal amount of information stored within the volume $r$ is

$$
N=\left(r / L_{*}\right)^{1+\frac{1}{\alpha}}
$$

This information hits its lower bound for gravity case, $\alpha=1$, and is unbounded for weaklycoupled non-classicalizing theories, that correspond to $\alpha=0$. More efficient is a classicalizer, more restrictive is the information storage within a given volume, and gravity provides an absolute bound.

Finally, notice that from (5.1) using (6.3) we can express the decay rate of any classicalon into few particles as

$$
\Gamma_{\text {classicalon } \rightarrow \text { few }} \sim \mathrm{e}^{-\left(r_{*} / L_{*}\right)^{1+\frac{1}{\alpha}}} .
$$

From here it is clear that among all classicalons, per fixed size, the black holes are the ones that decay into few particle states in a least suppressed way. This is because, among all the 
classicalons of a given size, the black holes are the ones that are made of the least number of soft quanta. This also explains, why for a weakly-coupled non-classicalizing theory $(\alpha=0)$ the above suppression is formally infinite. Because in such theories classicalons are never formed (even for energies corresponding to infinite $N$ ).

\subsection{Black hole entropy as classicalon entropy?}

In a quantum field theory framework the simplest way to assign an entropy to a black hole is to compute first the Hawking radiance temperature $T_{H}$ and to define the entropy by standard Clausius's rule: $T_{H}=\frac{\partial M}{\partial S_{b h}}$. This procedure leads to the well known black hole entropy $S_{b h}=\frac{A}{4}$ for $A$ being the horizon area in Planck length units. This relation between entropy and horizon area fits nicely with the black hole area theorems. The conceptual puzzle underlying the notion of black hole entropy is to understand its statistical meaning. Is this entropy the log of the number of internal black hole states associated with the same values of global mass, charge and angular momentum? Is the black hole entropy the log of the different ways we can create a given black hole? Is the entropy the log of the number of holographic quantum states we can fit on the horizon? Is it an entanglement entropy for the interior region? It is most likely, that all these questions can lead us to complementary understandings of the same fundamental issue.

On the other hand, as we tried to argue, the black hole formation can be viewed as a classicalization process, and black hole entropy has a precursor in classicalon language in terms of number of soft quanta produced in classicalization process. This fact may give us a possibility of pushing this connection further and understanding the origin of black hole entropy (at least qualitatively) in terms of classicalon states.

In other words, the notion of a classical interaction range $r_{*}$ leads to an alternative approach to black hole entropy and provides at least a qualitative way to define it in purely microscopic terms. The recipe is the following. Interpreting a black hole of mass $M$ as a non-perturbative state in the spectrum of pure Einsteinian gravity the associated entropy will be defined as the log of the number of quantum states of the set of the interacting quanta of wave-length $\sim r_{*}(M)$ that we can produce at energy $M$. The physical meaning of singling out such wavelengths is that this are dominant contributors in the classicalization process that takes place at distance $r_{*}(M)$, which defines the interaction range. Correspondingly, the number of these quanta is determined as,

$$
N(M)=M r_{*}(M)
$$

Therefore, the dimension of the corresponding Hilbert space of states will be $d=\xi^{N}$, for $\xi$ being a number of order one determined by the number of states of constituent quanta, such as e.g., helicities of the quanta of the theory. This leads to the entropy $S(M)=N(M) \ln (\xi)$. Hence we can estimate the black hole entropy once we know the classicalization radius of gravity. As shown in this paper the interaction range $r_{*}(M)$ for gravity is given by the Schwarzschild gravitational radius of $M$ and the previous recipe leads to a qualitatively-correct entropy counting. Note, that this approach to black hole entropy is purely statistical and does not use any notion of horizon or other geometric 
entities. The Clausius rule relating entropy and temperature appears as a consequence of the exponential suppression for the decay process of a set of $N$ "soft" quanta into a few "hard" ones. The rough estimate of this amplitude is

$$
\left(\frac{1}{M L_{P}}\right)^{N(M)}
$$

which agrees with the exponential suppression of (1.2).

The key point of the above approach to black hole entropy is, that it generalizes to any quantum field theory. Namely we can define an entropy function $S(M)$ depending on energy as $M r_{*}(M)$. Generically for renormalizable theories $r_{*}(M) \sim \frac{g}{M}$, where $g$ is some weak dimensionless coupling $g \ll 1$, and consequently $S(M) \sim 1$, i.e. no real notion of entropy emerges in such a case. Contrary, for theories that classicalize, i.e., for those with $r_{*}(M)$ growing as function of $M$ for $M \gg L_{*}^{-1}$, entropy starts to develop once we cross above the naive unitarity bound $M_{*}$. Note also, that this generalized notion of entropy for theories that classicalize always grows with energy.

\section{Conclusions}

The purpose of this paper is to establish close parallel between classicalization processes in gravity and in non-gravitational theories. For this, we have deconstructed the process of $s$-wave high energy scattering in gravity, which by all accounts is expecting to end up by black hole formation. For us, however, most important aspect was understanding of the pre-horizon-formation stage of this process from the point of view of classicalization. We have seen, that this process is nothing but a version of classicalization process that also takes place in analogous scattering of derivatively-coupled Nambu-Goldstone-type scalars.

The main finding of this analysis is, that $r_{*}(s)$ for gravity agrees with the gravitational radius of energy $\sqrt{s}$. This allows us to understand most of the black hole unitarization properties as direct consequences of classicalization.

In particular we see, that there is a clear precursor of entropy in terms of number of soft quanta produced in the classicalization process. This notion is universally applicable to generic classicalizing theories, regardless of the existence of the horizon, and follows from classicality of the configuration produced.

This universality allows for a parameterization of classicalization landscape in terms of a parameter that measures the classicalization-efficiency, and for generalization of black hole properties, such as holographic bound on information storage, to non-gravitational situations. Being a most efficient classicalizer, gravity is the limiting case on this landscape, and correspondingly, gives a most stringent bound on information storage.

\section{Acknowledgments}

We thank Gian Giudice for ongoing discussions and collaboration on classicalization. We thank Thibault Damour, Andrei Gruzinov, Cristiano Germani and Goran Senjanovic for discussions. The work of G.D. and C.G. was supported in part by Humboldt Foundation 
under Alexander von Humboldt Professorship, by European Commission under the ERC advanced grant 226371, and by the NSF grant PHY-0758032. The work of C.G. was supported in part by Grants: FPA 2009-07908, CPAN (CSD2007-00042) and HEPHACOSS2009/ESP1473. The work of A.K is supported by a PEVE-NTUA-2009 grand.

Open Access. This article is distributed under the terms of the Creative Commons Attribution Noncommercial License which permits any noncommercial use, distribution, and reproduction in any medium, provided the original author(s) and source are credited.

\section{References}

[1] G. Dvali, G.F. Giudice, C. Gomez and A. Kehagias, UV-Completion by Classicalization, arXiv: 1010.1415 [INSPIRE].

[2] G. Dvali and D. Pirtskhalava, Dynamics of Unitarization by Classicalization, Phys. Lett. B 699 (2011) 78 [arXiv:1011.0114] [INSPIRE].

[3] G. Dvali, Classicalize or not to Classicalize?, arXiv:1101.2661 [INSPIRE].

[4] G. Dvali and C. Gomez, Self-Completeness of Einstein Gravity, arXiv:1005.3497 [INSPIRE].

[5] G. Dvali, S. Folkerts and C. Germani, Physics of Trans-Planckian Gravity, Phys. Rev. D 84 (2011) 024039 [arXiv: 1006. 0984] [InSPIRE].

[6] B. Bajc, A. Momen and G. Senjanović, Classicalization via Path Integral, arXiv:1102.3679 [INSPIRE].

[7] K. Thorne, Black Holes and Time Warps: Einstein's Outrageous Legacy, W.W. Norton \& Company, New York U.S.A. (1995).

[8] G. 't Hooft, Graviton Dominance in Ultrahigh-Energy Scattering, Phys. Lett. B 198 (1987) 61 [InSPIRE].

[9] I. Muzinich and M. Soldate, High-Energy Unitarity of Gravitation and Strings, Phys. Rev. D 37 (1988) 359 [InSPIRE].

[10] D. Amati, M. Ciafaloni and G. Veneziano, Superstring Collisions at Planckian Energies, Phys. Lett. B 197 (1987) 81 [InSPIRE].

[11] D. Amati, M. Ciafaloni and G. Veneziano, Classical and Quantum Gravity Effects from Planckian Energy Superstring Collisions, Int. J. Mod. Phys. A 3 (1988) 1615 [InSPIRE].

[12] D. Amati, M. Ciafaloni and G. Veneziano, Higher order gravitational deflection and soft bremsstrahlung in planckian energy superstring collisions, Nucl. Phys. B 347 (1990) 550 [INSPIRE].

[13] D.J. Gross and P.F. Mende, String Theory Beyond the Planck Scale, Nucl. Phys. B 303 (1988) 407 [INSPIRE].

[14] S.B. Giddings, D.J. Gross and A. Maharana, Gravitational effects in ultrahigh-energy string scattering, Phys. Rev. D 77 (2008) 046001 [arXiv:0705.1816] [INSPIRE].

[15] D. Amati, M. Ciafaloni and G. Veneziano, Can Space-Time Be Probed Below the String Size?, Phys. Lett. B 216 (1989) 41 [inSPIRE].

[16] K. Konishi, G. Paffuti and P. Provero, Minimum Physical Length and the Generalized Uncertainty Principle in String Theory, Phys. Lett. B 234 (1990) 276 [InSPIRE]. 
[17] E. Witten, Reflections on the Fate of Spacetime, Phys. Today 49 (1996) 24.

[18] S.B. Giddings, M. Schmidt-Sommerfeld and J.R. Andersen, High energy scattering in gravity and supergravity, Phys. Rev. D 82 (2010) 104022 [arXiv: 1005.5408] [INSPIRE].

[19] S.B. Giddings, The gravitational S-matrix: Erice lectures, arXiv:1105.2036 [INSPIRE].

[20] M.W. Choptuik and F. Pretorius, Ultra Relativistic Particle Collisions, Phys. Rev. Lett. 104 (2010) 111101 [arXiv:0908.1780] [INSPIRE].

[21] P. D'Eath and P. Payne, Gravitational radiation in high speed black hole collisions. 1. Perturbation treatment of the axisymmetric speed of light collision, Phys. Rev. D 46 (1992) 658 [InSPIRE].

[22] P. D'Eath, Gravitational radiation in high speed black hole collisions, Class. Quant. Grav. 10 (1993) S207 [inSPIRE].

[23] H.-J. Matschull, Black hole creation in $(2+1)$-dimensions, Class. Quant. Grav. 16 (1999) 1069 [gr-qc/9809087] [INSPIRE].

[24] L. Blanchet and T. Damour, Hereditary effects in gravitational radiation, Phys. Rev. D 46 (1992) 4304 [INSPIRE].

[25] L. Blanchet, Quadrupole-quadrupole gravitational waves, Class. Quant. Grav. 15 (1998) 89 [gr-qc/9710037] [INSPIRE].

[26] L. Blanchet, Gravitational wave tails of tails, Class. Quant. Grav. 15 (1998) 113 [gr-qc/9710038] [INSPIRE].

[27] L. Blanchet, Gravitational radiation from post-Newtonian sources and inspiralling compact binaries, Living Rev. Rel. 9 (2006) 4 [gr-qc/0202016].

[28] R. Adler, J. Bjorken, P. Chen and J. Liu, Simple analytic models of gravitational collapse, Am. J. Phys. 73 (2005) 1148 [gr-qc/0502040] [InSPIRE].

[29] K. Thorne, Multipole Expansions of Gravitational Radiation, Rev. Mod. Phys. 52 (1980) 299 [INSPIRE].

[30] F. Berkhahn, D.D. Dietrich and S. Hofmann, Cosmological Classicalization: Maintaining Unitarity under Relevant Deformations of the Einstein-Hilbert Action, Phys. Rev. Lett. 106 (2011) 191102 [arXiv:1102.0313] [INSPIRE].

[31] C. Deffayet, X. Gao, D. Steer and G. Zahariade, From k-essence to generalised Galileons, Phys. Rev. D 84 (2011) 064039 [arXiv:1103.3260] [InSPIRE]. 